\section{Monitorização Contínua de Glicose: Análise Crítica Baseada em Experiência ao Longo de Um Ano}

\section{RESUMO}

A avaliação do controle glicêmico no diabetes mellitus (DM) envolve tradicionalmente a observação das taxas de glicemia e hemoglobina glicada. Recentemente o Fleury - Centro de Medicina Diagnóstica implantou o exame de monitorização contínua de glicose (MCG) (Medtronic Minimed - CGMS ${ }^{\circledR}$ System GoldTM) e, neste trabalho, objetivamos descrever a experiência relacionada à realização deste exame durante o ano de 2004. Realizaram-se 141 exames neste período. Do total, $88 \%(n=124)$ pacientes eram diabéticos, sendo 99 usuários de insulina. Encontramos forte correlação entre os valores de glicose obtidos com a MCG e no sangue capilar $(r=0,926 ; p<0,005)$. Nos diabéticos, identificou-se hipoglicemia noturna (<50mg/dL) em 35\% $(n=44)$, padrões hiperglicêmi$\cos (>220 \mathrm{mg} / \mathrm{dL}$ ) em períodos determinados do dia em $\sim 44 \%$ e hiperglicemia sustentada ao longo de toda monitorização em treze casos (10\%). Doze exames foram realizados para investigação de hipoglicemias em não diabéticos. Dois exames foram sugestivos de "dumping" e em um caso a MCG reforçou a hipótese de insulinoma. Ocorreram interrupções parciais das monitorizações em 15\% dos exames. Concluímos que a MCG é uma metodologia útil para investigação das oscilações glicêmicas, sendo uma importante ferramenta para ajuste terapêutico em pacientes com DM. (Arq Bras Endocrinol Metab 2005;49/6:983-990)

Descritores: Diabetes mellitus; Monitorização contínua de glicose; CGMS; Hiperglicemia; Hipoglicemia

\section{ABSTRACT}

\section{Continuous Glucose Monitoring: A Critical Appraisal After One Year Experience.}

Conventional assessment of glycemic control in diabetes mellitus (DM) includes blood glucose attention to glycemia and glycated hemoglobin levels. Recently, we introduced the continuous glucose-monitoring test (CGM) (Medtronic Minimed - CGMS ${ }^{\circledR}$ System Gold ${ }^{\mathrm{TM}}$ ). Here we describe our experience with this methodology over the year 2004. A total of 141 CGM tests were performed over this period of time. Overall, $88 \%(n=124)$ patients were diabetics (DM), 99 of them were insulin users. We found a strong correlation between glucose values obtained by CGM and capillary glucose measures $(r=0.926 ; p<0.005)$. In diabetic patients, nocturnal hypoglycemia ( $<50 \mathrm{mg} / \mathrm{dL}$ ) was identified in $\sim 35 \%(n=44)$, hyperglycemic patterns ( $>220 \mathrm{mg} / \mathrm{dL}$ ) at specific times of day in $\sim 44 \%$ and sustained hyperglycemia throughout the whole monitoring period in thirteen cases (10\%). Twelve tests were performed to investigate the occurrence of hypoglycemia in non-diabetic subjects. Two tests came out very suggestive of "dumping", and in one case the CGMS supported the hypothesis of insulinoma. Partial monitoring interruptions have occurred in $15 \%$ of all tests. We concluded that CGMS is a useful methodology to investigate glycemic fluctuations, and it is also an important tool to adjust therapy in diabetic patients. (Arq Bras Endocrinol Metab 2005;49/6:983-990)

Keywords: Diabetes mellitus; Continuous glucose monitoring; CGMS; Hyperglycemia; Hypoglycemia
Cláudia H.M.C. de Oliveiva Karina Berger Silvia Coral de A.L. e Souza Suemi Marui Cristina Khawali Omar M. Hauache José Gilberto H. Vieira Rui M.B. Maciel André F. Reis

Centro de Provas Funcionais e Núcleo Integrado de Diabetes, Fleury - Medicina Diagnóstica (CHMCO, KB, SM, CK, OMH, JGHV, RMBM, AFR);

Laboratório de Hormônios e Genética Molecular-LIM 42, Divisão de Endocrinologia e Metabologia, Hospital das Clinicas da Faculdade de Medicina da Universidade de São Paulo (SM, KB); e Laboratório de Endocrinologia Molecular, Disciplina de

Endocrinologia, Departamento de Medicina, Escola Paulista de Medicina, Universidade Federal de São Paulo (OMH, JGHV, RMBM, AFR), São Paulo, SP.

Recebido em 28/03/05 Revisado em 27/07/05 Aceito em 03/10/05 
$\mathbf{O}$ DIABETES MELLITUS (DM) apresenta elevada prevalência, sendo relacionado a um grande número de co-morbidades, cujo desenvolvimento e evolução encontram-se intimamente associados ao controle crônico das taxas glicêmicas. Entre os parâmetros laboratoriais freqüentemente utilizados para nortear as decisões terapêuticas voltadas ao controle glicêmico do DM, podemos citar a medida da glicemia de jejum, a frutosamina e a hemoglobina glicada (AlC). É importante salientar que os estudos prospectivos da associação entre níveis glicêmicos e complicações vasculares no DM basearam-se nas medidas de AlC (ex. DCCT e UKPDS), sendo este considerado o melhor indicador do controle glicêmico a médio-prazo (2-3 meses) (1-3). A auto-monitorização de glicemias capilares realizadas em glicosímetros de uso domiciliar é, por sua vez, um excelente recurso utilizado para retratar as flutuações glicêmicas ao longo do dia, sendo uma ferramenta bastante utilizada no acompanhamento dos pacientes com DM em esquema de tratamento intensivo, especialmente naqueles sob uso de múltiplas doses diárias de insulina.

Recentemente, um novo exame veio contribuir para a avaliação do controle glicêmico. A monitorização contínua da glicose subcutânea (MCG) (CGMS Continuous Glucose Monitoring System - MiniMed ${ }^{\circledR}$ Medtronic) permite acompanhar de maneira ininterrupta as variações das taxas glicêmicas, podendo ser uma metodologia auxiliar no acompanhamento de pacientes com DM, assim como em outras condições clínicas que cursam com oscilações dos níveis de glicemia. A experiência com este método é ainda limitada no Brasil.

O presente relato tem como objetivo descrever a experiência inicial com a MCG em caráter ambulatorial em um grupo heterogêneo de indivíduos e oferecer subsídios para a análise crítica da utilidade deste novo exame na prática clínica.

\section{MATERIAL E MÉTODOS}

No período de lo de janeiro de 2004 a 08 de fevereiro de 2005, 141 pacientes realizaram o exame de MCG no Laboratório Fleury - Centro de Medicina Diagnóstica, São Paulo, SP. Os exames foram realizados mediante solicitação dos médicos assistentes, totalizando 95 exames com duração de 72 horas, 21 com duração de 48 horas e 25 exames com duração de 24 horas. As indicações para a realização dos exames foram relacionadas à avaliação do controle glicêmico em diabéticos, suspeita de hipoglicemias, avaliação do controle glicêmico em diabéticas grávidas ou com diabetes gestacional, suspeita de insulinoma, suspeita de síndrome de dumping e uma minoria de outras indicações (ex.: oscilação glicêmica em usuário de glicocorticóide, avaliação de hiperglicemias em pré-diabéticos).

Os pacientes realizaram os seus exames em uma única unidade do Fleury, na cidade de São Paulo. Os exames de MCG foram realizados baseados no seguinte protocolo: após agendamento prévio, os pacientes comparecem ao laboratório levando o seu glicosímetro pessoal. Todos os atendimentos são realizados por uma endocrinologista e uma auxiliar de enfermagem, que, após fornecerem as orientações iniciais a respeito do exame, procedem à instalação do sensor de glicose subcutâneo. O sensor é fixado à pele do local escolhido para a sua inserção com uma tira de Micropore ou Tegaderm e conectado a um monitor de glicose (CGMS System Gold Monitor ${ }^{\circledR}$, Minimed, Inc.). Os pacientes permanecem no laboratório nos primeiros 60 minutos após a instalação do sensor, sendo que ao final desta primeira hora (período de "aquecimento do sensor") é realizada a glicemia capilar inicial (de ponta de dedo) para calibração do monitor de glicose. Enquanto aguarda, o paciente recebe orientações adicionais e um manual impresso com informações mais detalhadas sobre como proceder durante o período de monitorização. Solicita-se que os pacientes façam no mínimo duas e idealmente quatro glicemias capilares diárias, não ultrapassando um período de 12 horas de intervalo entre cada uma delas. Os pacientes são orientados a registrar estes valores de glicemia diariamente no monitor de glicose e também anotá-los em um diário pessoal, onde outras informações relevantes também são relatadas, tais como sintomas de hipoglicemia, doses de insulina, mudanças dietéticas, atividade física e outras. Ressalta-se a importância da manutenção das atividades habituais, com exceção das atividades aquáticas (como hidroginástica, natação), devendo o monitor ser protegido por um invólucro plástico apropriado (Shower-Pak ${ }^{\circledR}$ Minimed) durante o banho.

O monitor armazena até 288 medidas de glicose intersticial por dia de exame. Após a finalização do teste, estes dados são capturados para uma base de comunicação (Com-Station, Minimed $\left.{ }^{\circledR}\right)$ e transportados para um software (Minimed Solutions CGMS Software ${ }^{\circledR}$ Minimed, Inc.) que faz a análise gráfica e estatística dos valores obtidos. Ao final, produz-se um relatório resumindo as informações mais relevantes: os valores glicêmicos diários (máximo, mínimo e média) e no período total da monitorização, um laudo interpretativo sintético, e a apresentação de gráficos que ilustram as 
medidas de glicemia de cada dia de monitorização e de todos os dias do exame sobrepostos para a avaliação das tendências glicêmicas diárias. Quando informativo, gráficos ampliando períodos específicos do dia são gerados para salientar uma ocorrência relevante e com padrão repetitivo (por exemplo, hipoglicemia noturna, elevações glicêmicas pós-prandiais).

As medidas de glicemia capilar foram realizadas pelos pacientes em diferentes glicosímetros pessoais (One Touch, Accucheck, Advantage, Optium) calibrados de acordo com as instruções do fabricante. Para garantir maior especificidade no diagnóstico das hipoglicemias, foi considerado um valor de corte de $\leq$ $50 \mathrm{mg} / \mathrm{dL}$. Pela mesma razão, considerou-se hiperglicemia quando os valores estiveram acima de $220 \mathrm{mg} / \mathrm{dL}$. Foram considerados para análise os valores de hemoglobina glicada realizados no Fleury, no período máximo de seis semanas anteriores à realização da MCG até quatro semanas após a realização da mesma (disponíveis em 60 pacientes). O método para dosagem da hemoglobina glicada no laboratório Fleury é Cromatografia Líquida de Alta Performance (HPLC), com valor de referência de 4,0-6,0\%.

\section{Análise estatística}

As variáveis de distribuição normal foram apresentadas como média e desvio-padrão e as não-paramétricas como mediana e valores mínimo e máximo. A análise de correlação entre as variáveis pareadas (Glicemia capilar média, Glicemia intersticial média e AlC) foi realizada pelo cálculo do coeficiente de Pearson e regressão linear.

\section{RESULTADOS}

Foi realizado um total de 141 exames neste período de um ano. Os dados demográficos dos indivíduos estudados encontram-se na tabela 1 . Nota-se que a maior parte dos exames foi feita em pacientes com DM (88\% - 124 pacientes) e cinco indivíduos realizaram o exame devido à suspeita de diabetes. Uma boa parcela dos diabéticos estava em uso de insulina (99 pacientes), dentre eles oito pacientes sob uso de bomba de infusão contínua de insulina. Quatro pacientes eram gestantes, sendo duas previamente diabéticas e duas pacientes com diabetes gestacional em curso. Entre as indicações mais freqüentemente citadas para a realização do exame nos diabéticos destacam-se a avaliação de hipoglicemia (25 pacientes), discrepância entre AlC e glicemias capilares, instabilidade glicêmica e avaliação após mudança de esquema terapêutico.

A análise comparativa entre os valores glicêmicos médios diários de todos os indivíduos mostrou forte correlação entre as medidas de glicose subcutânea obtidas pela MCG e as glicemias capilares obtidas pelas medidas de ponta de dedo $(\mathrm{r}=0,926$; $\mathrm{p}<$ $0,005)$, conforme indicado na figura 1 . O mesmo tipo de análise envolvendo as medidas de $\mathrm{AlC}$ e as glicemias médias de ponta de dedo, e as médias das medidas de glicose obtidas pela MCG mostrou um coeficiente de correlação respectivamente de 0,582 e $0,603(\mathrm{p}<0,005)$ (figuras $2 \mathrm{~A}$ e $2 \mathrm{~B})$.

\section{Avaliação de hiperglicemias nos diabéticos}

Do total de diabéticos, $42 \%$ ( 52 pacientes) mantiveram hiperglicemia $(\geq 220 \mathrm{mg} / \mathrm{dL})$ por mais de $25 \%$ do tempo total da monitorização. Treze pacientes $(10 \%$ dos diabéticos) apresentaram hiperglicemia mantida durante todo tempo de exame. Adicionalmente, a observação gráfica das curvas de MCG revelou padrões de hiperglicemia concentrada em períodos específicos do dia em $44 \%$ dos diabéticos examinados.

\section{Avaliação de hipoglicemias nos diabéticos}

A avaliação dos valores diários de glicose intersticial obtidos pela MCG revelou a ocorrência de pelo menos um evento hipoglicêmico $(\leq 50 \mathrm{mg} / \mathrm{dL})$ em $54 \%$ dos pacientes diabéticos $(n=67)$. Em 35\% $(n=44)$ dos diabéticos foram detectados episódios hipoglicêmicos durante o período noturno e a madrugada.

Tabela 1. Características dos pacientes submetidos ao exame de MCG no Laboratório Fleury.

\begin{tabular}{lc}
\hline Total & 141 indivíduos \\
\hline Idade (anos) & $43,6 \pm 19,8^{*}$ (intervalo: 9 meses a 91 anos) \\
IMC $\left(\mathrm{Kg} / \mathrm{m}^{2}\right)$ & $24,74 \pm 4,56^{*}$ \\
Sexo & $69 \mathrm{M}, 72 \mathrm{~F}$ \\
Diabético* & 124 indivíduos $(88 \%)$ \\
Uso de insulina & 99 diabéticos $(8$ em sistema de infusão contínua) \\
AlC $(\%)$ & $7,95 \pm 1,66^{*}(\mathrm{n}=60)$ \\
Gestantes & $4(1 \mathrm{DM1}, 1 \mathrm{DM} 2,2 \mathrm{DM}$ gestacional $)$ \\
\hline
\end{tabular}

* Média \pm DP (intervalo)

** A separação entre DM1 e DM2 não foi realizada devido à insuficiência de dados clínicos e laboratoriais para estabelecer o diagnóstico diferencial. 


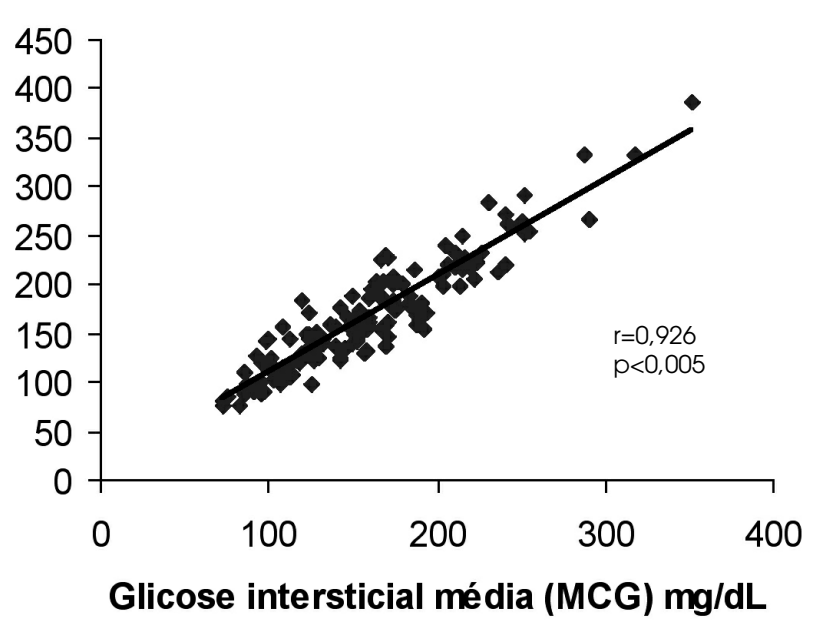

Figura 1. Correlação entre valores de glicose intersticial medidos pela MCG e valores de glicose capilar (de ponta de dedo) medidos em glicosímetros.
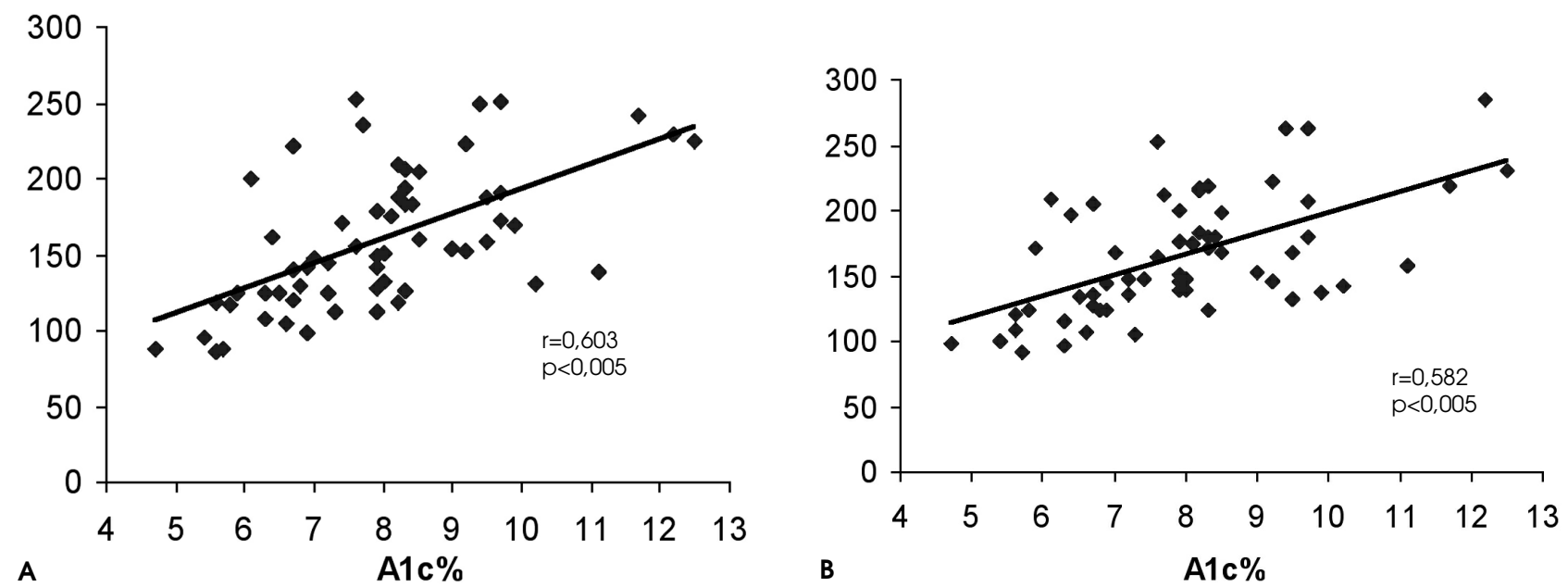

Figura 2. Correlação entre medidas de hemoglobina glicada (A1C) e valores de glicose medidos pela MCG (figura 2A) e valores de glicose capilar medidos em glicosímetros (figura 2 B).

Em um pequeno grupo de diabéticos $(n=9$; $8 \%$ ), o número de medidas de glicose abaixo de $50 \mathrm{mg} / \mathrm{dL}$ atingiu mais de $20 \%$ do tempo total do exame. Entre estes nove indivíduos, encontravam-se pacientes com bom controle glicêmico (AlC $<6,9 \%$ em 4 casos) mas também alguns indivíduos com controle metabólico diurno insatisfatório ( 3 pacientes com elevadas médias de tempo em hiperglicemia: 19\%, 29\% e $37 \%$ do tempo total de monitorização).

Cinco pacientes realizaram o exame em dois momentos diferentes, antes e após mudanças terapêuticas que resultaram da interpretação do exame inicial. Em um deles, a primeira MCG evidenciou um padrão gráfico repetitivo de tendência à hipoglicemia durante $o$ período noturno, de duração prolongada e assintomáti- ca. Após o ajuste das doses de insulina diárias, o exame foi repetido revelando uma diminuição sensível no número de eventos hipoglicêmicos noturnos (figura 3).

\section{Avaliação de hipoglicemias em não-diabéticos}

Doze indivíduos não-diabéticos foram avaliados devido à suspeita de hipoglicemia. Em sete deles, a MCG detectou pelo menos um episódio de hipoglicemia. Entretanto, a correlação entre os sintomas relatados (por exemplo: mal-estar, sudorese, tremores, fraqueza, tonturas) e os níveis de glicemia demonstrados pela MCG foi muito pobre, exceto para três pacientes, descritos a seguir. A avaliação das curvas diárias de glicose ao longo da MCG sugeriu a hipótese de dumping em dois pacientes cujo histórico envolvia um passado

Arq Bras Endocrinol Metab vol 49 nº 6 Dezembro 2005 

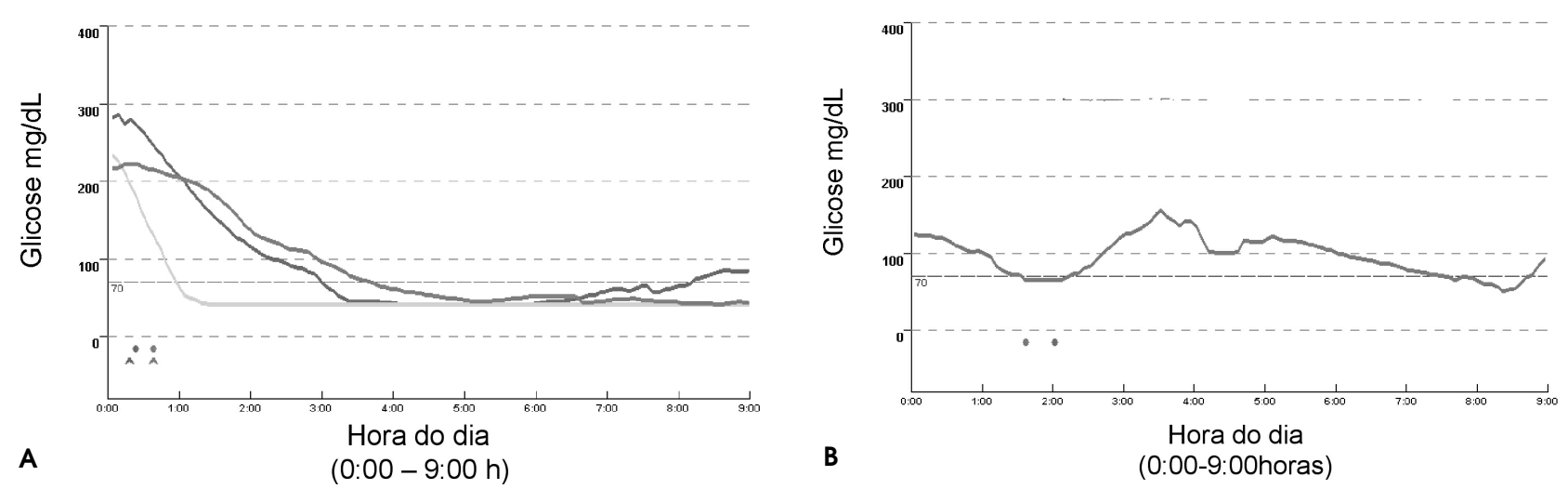

Figura 3. Apresentação de um caso de hipoglicemia noturna pré e pós ajuste terapêutico baseado na MCG. Figura 3A Paciente M.H.M, masculino, 45 anos. Ampliação das curvas de MCG no período noturno (0:00 a 9:00h) de três dias consecutivos. Figura 3B - Exemplo da MCG do mesmo paciente em uma noite típica na repetição do exame após mudança de tratamento (os círculos cheios indicados nos gráficos correspondem ao registro de uma refeição realizada pelo paciente e as pontas de seta indicam ao momento de uma aplicação de insulina).

de cirurgia gástrica. Um destes pacientes era uma criança de 8 meses de idade, alimentada por meio de gastrostomia, internada em UTI pediátrica, apresentando hipoglicemias freqüentes e convulsões de etiologia não esclarecida. Neste caso, o padrão glicêmico diário demonstrado pela MCG foi bastante característico da síndrome de dumping. A outra paciente, de 49 anos de idade, apresentava queixas de mal-estar e síncopes pósalimentares freqüentes, e apresentava um histórico de cirurgia bariátrica há 2 anos. Estes dois casos estão ilustrados na figura 4. Entre dois pacientes cuja indicação do exame baseava-se em uma hipótese de insulinoma, um caso mostrou padrão glicêmico diário sugestivo de hipoglicemias pós-absortivas. Esta paciente com 70 anos, não-diabética, com história de episódios hipoglicêmicos freqüentes, acompanhados de perda de consciência e convulsões, chegou a apresentar valores glicêmicos abaixo de $50 \mathrm{mg} / \mathrm{dL}$ durante $22 \%$ e $25 \%$ do tempo durante dois dos três dias de monitorização.

\section{Reaçōes adversas e intercorrências}

As reações adversas mais freqüentemente observadas foram: irritação cutânea no local do curativo (hiperemia/pápula) em 6 casos ( 4\%), presença de leve sangramento no sítio de inserção do sensor em 6 casos $(\sim 4 \%)$, dor persistente após a instalação do sensor em 2 casos $(1,4 \%)$. Síncope vaso-vagal ocorreu em 2 casos $(1,4 \%)$, um imediatamente após a inserção do sensor, e outro cerca de 20 minutos após o mesmo. Neste último caso foi descartada a hipótese da hipoglicemia.

Em cerca de $15 \%$ dos exames (2l casos) ocorreram interrupções parciais da monitorização atribuídas, na sua maioria, a problemas técnicos. Estas interferências em parte deveram-se ao uso incorreto do apa- relho pelo paciente tais como dificuldade nos registros das glicemias capilares, demora de mais de 12 horas entre duas medidas de glicemia capilar, acidentes com o monitor. Outras causas identificadas foram defeitos no sensor de glicose, alterações cutâneas no local de instalação do sensor e outras de origem não identificada. O exame foi considerado inválido se o período de interrupção comprometesse a sua interpretação global. Assim, em quatro casos $(\sim 3 \%)$ foi necessária a repetição do mesmo por total impossibilidade de interpretação dos resultados.

\section{DISCUSSÃO}

Na nossa experiência, a MCG mostrou-se útil para detecção de hipoglicemias e hiperglicemias em uma boa parcela dos casos encaminhados. Em uma minoria dos exames notaram-se intercorrências principalmente relacionadas à interrupção parcial da monitorização devido a falhas na manipulação do sistema.

$\mathrm{O}$ enfoque atual no controle glicêmico objetiva não somente evitar a ocorrência das complicações agudas e complicações vasculares associadas à hiperglicemia, mas também minimizar a ocorrência de hipoglicemias e seus riscos, priorizando ainda a flexibilidade e a qualidade de vida do paciente. Dentro deste contexto, exames que permitam a monitorização contínua da glicemia ao longo das 24 horas do dia assumem um valor claro ao orientar ajustes de doses e de medicações visando atingir as metas glicêmicas ótimas e seguras estabelecidas.

A experiência inicial com a MCG aqui descrita demonstrou a utilidade desta metodologia como 

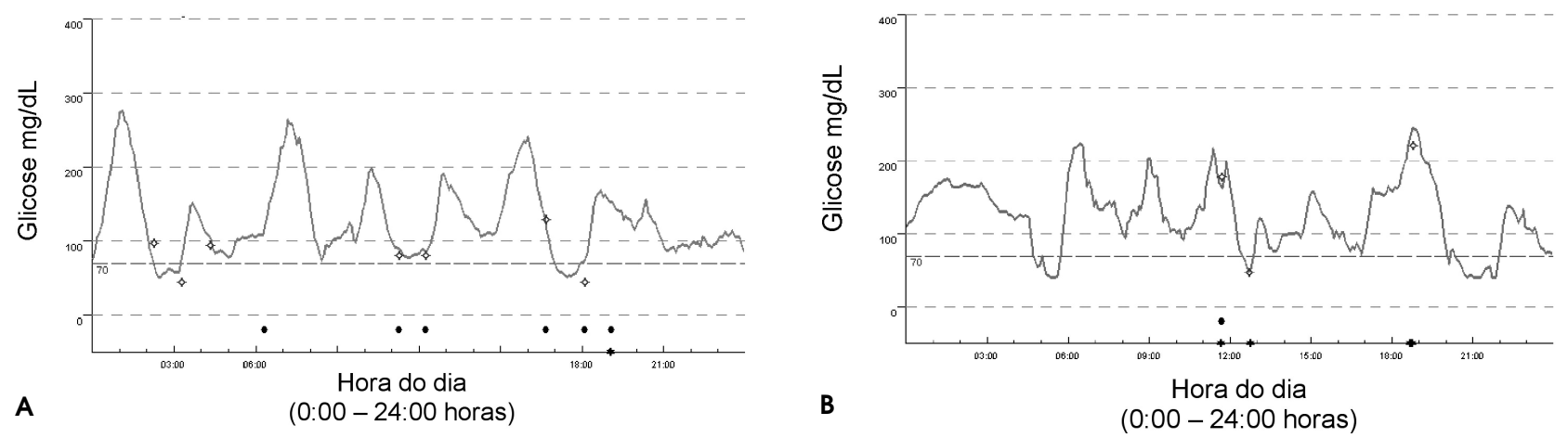

Figura 4. Apresentação de dois casos sugestivos de "dumping". Figura 4A - Paciente P.R.G.P., masculino, 9 meses de idade, gastrostomia, hipoglicemias freqüentes e convulsões. Figura 4B - Paciente C.M.G., feminina, 49 anos de idade, histórico de cirurgia bariátrica, mal-estar e síncopes pós-alimentares (os losangos vazados presentes nas curvas de MCG correspondem às glicemias capilares registradas durante o exame. Os círculos cheios abaixo das curvas dos gráficos indicam o registro do início de uma refeição realizada pelo paciente e os asteriscos na linha de base da linha do tempo correspondem a diferentes eventos registrados pelos pacientes).

potencial ferramenta para orientar a melhoria do controle glicêmico dos pacientes com DM encaminhados para a realização do exame. Apesar de reconhecermos que muitos casos foram encaminhados por apresentarem algum grau de dificuldade de controle, a casuística aqui apresentada retrata as grandes oscilações glicêmicas apresentadas pelos diabéticos, em especial naqueles utilizando insulina. A análise dos padrões de hiperglicemia das curvas da MCG forneceu subsídios para o ajuste de dose de medicamentos, principalmente nos usuários de análogos de insulina de ação rápida ou de bomba de infusão contínua.

Foi notável na nossa experiência a elevada incidência de hipoglicemias entre os pacientes diabéticos (35\% do pacientes apresentando hipoglicemias noturnas), mesmo em usuários de hipoglicemiantes orais. Destaca-se o fato de que a grande maioria dos episódios hipoglicêmicos observados, principalmente os noturnos, foi assintomática e de duração prolongada. A inconveniência da realização da glicemia de ponta de dedo na madrugada, somada à freqüente falta de percepção da hipoglicemia noturna (especialmente em diabéticos com vários anos de diagnóstico), pode ocasionar uma subestimação do número de episódios de hipoglicemias pelos métodos de auto-monitorização habituais, sendo os mesmos revelados pelo exame de MCG. Por outro lado, estudos publicados indicam que os métodos atuais de avaliação contínua de glicemia apresentam uma performance inferior na faixa de hipoglicemia, comparada aos valores glicêmicos elevados $(4,5)$. Deste modo, não podemos deixar de considerar que, especialmente no que tange à hipoglicemia assintomática em diabéticos, $(6,7)$, a freqüência de hipoglicemia poderia estar sendo de alguma forma superestimada, quando se consideram somente o número de episódios hipoglicêmicos. Contudo, na nossa experiência, a observação gráfica das curvas da MCG, indicando uma tendência evidente à queda dos níveis de glicose para abaixo dos níveis fisiológicos, foi o achado principal que motivou uma descrição da hipoglicemia na interpretação do exame, sendo mais relevante do que a observação de valores hipoglicêmicos isolados. Vale ressaltar, ainda, que a indicação para o exame de MCG em vários destes pacientes baseouse na suspeita clínica de hipoglicemia, reforçando os achados da monitorização. A revelação destas curvas de tendências mostrou-se o melhor indicador da necessidade de uma intervenção terapêutica, resultando em benefícios para o paciente, conforme demonstrado na figura 3 .

A correlação observada entre as medidas de glicose capilar e as medidas de glicose obtidas pelo sensor foi excelente e consistente com a literatura (8). Uma vez que os valores de glicose intersticial extraídos da MCG sofrem influência da freqüente calibração do monitor pelas medidas de glicemia capilar, uma elevada correlação entre estas duas variáveis é esperada e reflete apenas o controle interno de qualidade do exame. A correlação entre as medidas da MCG e as medidas de glicose sangüínea pelo método de referência não foi demonstrada no presente estudo e é considerada bastante satisfatória conforme demonstra a literatura, o que justificou a validação do método. É importante ressaltar, no entanto, que a avaliação das medidas de glicemia obtidas através da MCG é retrospectiva, não sendo possível a visualização destes valores em tempo real. As concentrações de glicose medidas no líquido intersticial refletem as oscilações gli- 
cêmicas ocorridas no fluido intravascular com um atraso de 5 a 10 minutos (9). Estas medidas intersticiais também sofrem a influência de variações na sensibilidade à insulina. Já foi descrito que em situações de incremento rápido da glicose sangüínea (por exemplo, sobrecarga endovenosa de glicose), a elevação das medidas de glicemia obtidas através do sensor subcutâneo é 30\% inferior às medidas realizadas no sangue, refletindo possivelmente o aumento da captação periférica de glicose (10). Por outro lado, em hipoglicemias induzidas pela administração de insulina endovenosa, a queda na glicose intersticial é também menos acentuada do que a observada no sangue, indicando ocorrer uma supressão no transporte da glicose do tecido intersticial. Resultados semelhantes foram demonstrados utilizando-se outra metodologia de medida da glicose do líquido intersticial (GlucoWatch ${ }^{\circledR}$ - Cygnus, Redwood City, CA, EUA) pela aplicação de uma corrente elétrica transcutânea, apresentando também um retardo de cerca de 15 minutos na detecção das variações glicêmicas (11). Desta forma, mediante hiper ou hipoglicemias agudas, decisões clínicas imediatas devem sempre ser tomadas baseadas nas glicemias capilares medidas pelo paciente quando indicado. Por esta razão, as medidas de glicemia capilar são ainda consideradas o meio mais confiável de avaliação das oscilações glicêmicas imediatas. Até que novos dispositivos de medida de glicose intersticial em tempo real estejam aprovados para o uso clínico (12), o exame de MCG na forma que se encontra atualmente disponível constitui-se em ferramenta laboratorial para complementar as informações obtidas pelos outros parâmetros de avaliação do controle glicêmico em diabéticos, não prescindindo da auto-monitorização contínua domiciliar.

Observamos, também, na nossa experiência uma excelente aceitação da técnica por parte dos pacientes e um alto grau de envolvimento com a rotina do exame. Apesar disto, em 15\% dos casos houve intercorrências que prejudicaram parcialmente a avaliação do exame. A taxa de intercorrências neste exame é em geral elevada, com índices de falha atingindo até $30 \%$ dos sensores inseridos (13). Este elevado índice de falhas operacionais ressalta a importância da boa seleção dos pacientes a serem submetidos ao exame, salientando-se que a melhor utilidade da MCG se manifesta naqueles pacientes cuja indicação for a mais precisa. Interessante salientar que em nossa experiência este índice sofreu uma redução gradual ao longo do tempo $(\sim 15 \%$ de interrupções parciais e 3\% de insucesso levando à repetição da MCG) provavelmente devido a maior treinamento dos profissionais envolvidos $\mathrm{e}$ fornecimento de maiores informações aos pacientes (14). Embora seja um número minoritário, 35\% dos pacientes não faziam uso de insulina ou não eram diabéticos, não estando habituados a realizar medidas de glicemia capilar, sendo que estes indivíduos apresentaram maior dificuldade em utilizar o glicosímetro e operar o monitor. Não obstante, em muitos casos, a oportunidade da realização de várias glicemias diárias em diferentes momentos do dia proporcionou aos pacientes menos adeptos desta prática o conhecimento da variabilidade diária de suas taxas glicêmicas sujeita às mudanças de cardápio, atividade física e efeitos das medicações hipoglicemiantes ou insulina. No caso de usuários de bomba de insulina, a avaliação dos perfis glicêmicos diários forneceu informações possivelmente úteis para o ajuste das doses de insulina basal e melhor adequação dos bolus de insulina pré-prandiais.

Finalmente, em relação aos encaminhamentos por hipoglicemias de causas mais raras, o uso da MCG revelou-se útil para confirmar o diagnóstico de dois casos de dumping. Por outro lado, em nenhum dos outros casos em que a suspeita clínica era de hipoglicemia reativa foi documentada uma associação entre a ocorrência dos sintomas e medidas de glicemia abaixo dos níveis normais. A possibilidade de avaliação das variações glicêmicas espontâneas destes pacientes e em sua rotina alimentar habitual, sem necessidade de um teste prolongado de sobrecarga oral com glicose, tornaria esta técnica bastante atraente para o auxílio diagnóstico de hipoglicemias de várias etiologias. No entanto, considerando-se as limitações do método em valores glicêmicos abaixo de $60 \mathrm{mg} / \mathrm{dL}$ (15), especialmente em indivíduos normais, esta metodologia necessita ainda ser validada como um recurso confiável para o diagnóstico de hipoglicemias em não diabéticos.

Em conclusão, a nossa experiência inicial com o sistema de MCG sugere que esta metodologia pode ser útil para auxílio de ajuste terapêutico em pacientes com DM, sendo também um exame auxiliar na confirmação da hipótese de síndrome de dumping. Este novo exame mostrou ser uma metodologia que pode trazer importantes informações para alguns casos de DM que apresentam dificuldades de controle glicêmico e hipoglicemias em períodos específicos do dia.

\section{AGRADECIMENTOS}

Os autores agradecem à equipe de atendimento do Laboratório Fleury pela colaboração na realização dos exames. 


\section{REFERÊNCIAS}

1. The Diabetes Control and Complications Trial Research Group. The effect of intensive treatment of diabetes on the development and progression of long-term complications in insulin-dependent diabetes mellitus. N Engl J Med 1993:329:977-86.

2. UK Prospective Diabetes Study (UKPDS) Group. Intensive blood-glucose control with sulphonylureas or insulin compared with conventional treatment and risk of complications in patients with type 2 diabetes (UKPDS 33). Lancet 1998;352:837-53.

3. UK Prospective Diabetes Study (UKPDS) Group. Effect of intensive blood-glucose control with metformin on complications in overweight patients with type 2 diabetes (UKPDS 34). Lancet 1998;352:854-65.

4. The Diabetes Research in Children Network (DirecNet) Study Group. Accuracy of the GlucoWa5tch $G 2$ biographer and the continuous glucose monitoring system during hypoglycemia. Diabetes Care 2004;27:722-6.

5. Klonoff DC. The need for separate performance goals for glucose sensors in the hypoglycemic, normoglycemic and hyperglycemic ranges. Diabetes Care 2004:27:834-6.

6. McGowan K, Thomas W, Moran A. Spurious reporting of nocturnal hypoglycemia by CGMS in patients with tightly controlled type 1 diabetes. Diabetes Care 2002;25:1499-503. (Errata em: Diabetes Care 2003; 26:553).

7. Conrad SC, Mastrototaro JJ, Gitelman SE. The use of a continuous glucose monitoring system in hypoglycemic disorders. J Pediatr Endocrinol Metab 2004:17:281-8.

8. Gross TM, Bode BW, Einhorn D, Kayne DM, Reed JH, White NH, et al. Performance evaluation of the MiniMed continuous glucose monitoring system during patient home use. Diabetes Technol Ther 2000:2:49-56.

9. Boyne MS, Silver DM, Kaplan J, Saudek CD. Timing of changes in interstitial and venous blood glucose measured with a continuous subcutaneous glucose sensor. Diabetes 2003:52:2790-4
10. Aussedat B, Dupire-Angel M, Gifford R, Klein JC, Wilson $G S$, Reach $G$. Interstitial glucose concentration and glycemia: implications for continuous subcutaneous glucose monitoring. Am J Physiol Endocrinol Metab 2000;278:E716-28.

11. Kulcu E, Tamada JA, Reach G, Potts RO, Lesho MJ. Physiological differences between interstitial glucose and blood glucose measured in human subjects. Diabetes Care 2003:26:2405-9.

12. Bode B, Gross K, Rikalo N, Schwartz S, Wahl T, Page C, et al. Alarms based on real-time sensor glucose values alert patients to hypo- and hyperglycemia: the guardian continuous monitoring system. Diabetes Technol Ther 2004:6:105-13.

13. Sachedina N, Pickup JC. Performance assessment of the Medtronic-MiniMed Continuous Glucose Monitoring System and its use for measurement of glycaemic control in Type 1 diabetic subjects. Diabet Med 2003:20:1012-5.

14. Sadler CE, Einhorn D. Office logistics: implementation of the continuous glucose monitoring system in clinical practice. Diabetes Technol Ther 2000;2(suppl. 1):S89-91.

15. Mauras N, Beck RW, Ruedy KJ, Kollman C, Tamborlane WV, Chase HP, et al. Diabetes Research in Children Network (DirecNet) Accuracy Study. Lack of accuracy of continuous glucose sensors in healthy, nondiabetic children: results of the Diabetes Research in Children Network (DirecNet) accuracy study. J Pediatr 2004; 144:770-5.

\section{Endereço para correspondência:}

André F. Reis

Núcleo Integrado de Diabetes, Fleury -

Medicina Diagnóstica

Av. Gal. Waldomiro de Lima 508

04344-070 São Paulo, SP

E-mail: andre.reis@fleury.com.br 